\title{
SPRÜCHE, SENTENZEN, ZITATE ALS KURZFORMEN ODER "EINFACHE FORMEN" DER TEXTSORTEN IN INTERKULTURELLER SICHT
}

\section{Einleitung}

Eine systematische sprachliche Behandlung der Sprichwörter in bezug auf die verschiedenen Sprachen und auf die sprachliche Varianz nur einer Sprache wäre eine umfangreiche Arbeit. Man kann aber doch in einem Beitrag, wo zwei oder drei Sprachen in Betracht gezogen werden, zuerst einige allgemeine Feststellungen erwähnen, womit die Varianz, die in den Bereich der Sprachwissenschaft gehört, einigermaßen prinzipiell zu lösen wäre und etliche Details der Stilistik (auch im ästhetischen Sinn) gezeigt werden können.

1. Die logische Grammatik, die man beherrschen muß, und die unterschiedlichen Akzeptabilitätsbedingungen, welche in bezug auf eine Gesamtsprache und -wenn notwendig - auch auf eine Sprachvariante (eines Diasystems) vorkommen, sind für das Verstehen der Sprüche, für die Konnotation ausschlaggebend.

Aber das darf nicht auf die Wahl einer Subgrammatik wirken bzw. die Sprecher-Hörer-Relation beschränken, sondern alles soll sich im Prinzip auf alle Regeln - die Einsetzungsregeln der Lexikoneinheiten mit eingerechnet - beziehen, daß man sieht, wie deren Verwendung unterschiedlichen Akzeptabilitätsbedingungen unterliegt.

2. Die pragmatischen Zeit- und Ortsbestimmungen werden aus dem pragmatischen Bereich in die Allgemeingültigkeit übertragen:

Heute rot, morgen tot.

Was du heute kannst besorgen, das verschiebe nicht auf morgen.

Wie man in den Wald hineinruft, so schallt es wieder heraus.

3. Die konnotativen Komponenten gehören zum Beschreiben dieses Typs, dieser Textsorte. Die Einführung der konnotativen Komponenten führt zum grammatischen und metagrammatischen Vergleich der verschiedenen Sprachen. Das kann für die Lexikologie als auch für die Textinterpretation und für die Stilistik von Bedeutung sein. Wir stellen die verschiedenen Subgrammatiken auf, deren obligatorische Regeln und allgemein verwendete Vokabeln mit der 
entsprechenden Leitkonnotation zusammenhängen, die fakultativen darüber hinaus mit den konnotativen Komponenten.

4. Man muß wissen, in welchen Grammatiken und Subgrammatiken gewisse Regeln vorkommen können.

5. Die im syntaktischen Bereich auftretenden Transformationen sind immer mit dem phonologischen Material verbunden. Die Veränderungen der Lautgestalt, die rhetorische Erscheinungen sind, beweisen, daß es sich beim Spruch um rhetorische Absichten handelt.

6. Reduktionen oder Ellipsen finden wir in den Sprüchen oft:

Morgenstund' hat Gold im Mund

Fleiß'ge Hand, gutes Land.

Wie gewonnen, so zerronnen.

7. Es geht bei den Sprüchen nicht um die Formulierung einer neuen Grammatik, sondern um die Bereicherung des gewählten Sprachsystems, oft durch neue Ausdrucksmöglichkeiten, die in diesem System prinzipiell möglich sind, wenngleich sie bei seiner normalen Verwendung de facto nicht oder nur sehr selten benutzt werden.

Viele von den Sprüchen können als rhetorisch bezeichnet werden; sie müssen auch so expliziert werden.

Die Regeln der Kompetenz können nicht den grammatischen Regeln gleichgesetzt werden. Es handelt sich bei den Kurzformen trotzdem nicht nur um Sprechsorten, sondern auch um Textsorten, die aber auch autorenspezifisch sind. Deswegen handelt es sich auch um die Stilistik. Die spezifischen rhetorischen Erweiterungen des grammatischen Systems sind mit der spezifischen Kompetenz verbunden. Die rhetorischen Regeln müssen sich aber mit den grammatischen doch im Einklang befinden und die grundlegenden Gesetze des grammatischen Systems befolgen.

Die Anwendungen verschiedener rhetorischer Regeln sind meist keine Verstöße gegen grammatische Regeln, sondern es sind neuartige Formulierungen, die auf Grund der Sprachkompetenz interpretierbar und im Prinzip möglich sind. ${ }^{1}$

Es ist nicht immer der Fall, daß den auf denselben sprachlichen Merkmalen beruhenden Relationen jeweils der gleiche poetische Wert zugeschrieben wird. ${ }^{2}$

$$
\begin{gathered}
\text { Ein zum populärsten Spruch gewordener } \\
\text { Grundgedanke und seine kontextuellen } \\
\text { Abwandlungen }
\end{gathered}
$$

Schauen wir uns einen Spruch an, der auch als Sentenz und Zitat betrachtet werden kann und der in drei Sprachen verschiedene Formen des Stils im breiteren Sinn

1 Zoltán Kanyo: Sprichwörter - Analyse einer einfachen Form. Ein Beitrag zur generativen Poetik. Budapest. Akadémiai Kiado 1981, S. 214.

2 Ebenda, S. 216. 
aufweist. Es gibt in den drei Sprachen jeweils verschiedene Wortkategorien, verschiedene grammatische Erscheinungen in den morphophonologischen sowie in den syntaktischen Strukturen, und somit gibt es auch in Details verschiedene Konnotationen. So entstehen auch unterschiedliche oder modifizierte soziokulturelle Beziehungen zur semantischen Struktur des Originals, das den jeweiligen Angehörigen der beiden anderen Sprachgemeinschaften vielleicht überhaupt nicht bekannt ist (bekannt sein kann oder zu sein braucht).

Hesiod schrieb einen kurzen Text, der aus drei Aussagen besteht, von denen jede als ein selbständiger Spruch oder eine selbständige Sentenz, als eine sog. einfache Textsorte aufgefaßt werden kann und auch für sich selbst stehen könnte.

So lautet der kurze Text in deutscher Übersetzung: Morgenfrühe gewährt immer ein Drittel vom Tagwerk; / Morgenfrühe verkürzt den Weg, macht kürzer die Arbeit. -Hesiod, Werke und Tage, $578 .^{3}$

Der lateinische Spruch Aurora Musis amica wird sehr häufig verwendet.

In einigen deutschen Zitatensammlungen, Lexiken, Wörterbüchern, Sprichwörtersammlungen usw. wird der lateinische Spruch unterschiedlich übersetzt, z.B. Die Morgenstunde ist den Musen günstig. Mit der Anführung des lateinischen Sprichwortes Aurora Musis amica in der Klammer. ${ }^{4}$

Man findet für den Spruch Aurora Musis amica auch diese Variante der Übersetzung: Die Morgenröte ist den Musen hold. Es ist interessant, daß es dabei noch einen neuen Hinweis gibt: "Entsprechend dem deutschen Morgenstund' hat Gold im Mund." Bezeichnet ist auch die Ellipse. ${ }^{5}$

Im Grimmschen Wörterbuch wird zuerst das deutsche Sprichwort morgenstund hat gold im mund angeführt, dann kommt die lateinische Form Aurora musis amica. Für den lateinischen Spruch wird als Quelle Pistorius thes. par. 6,6 genannt. ${ }^{6}$

Interessant ist eine etwas willkürliche Erklärung des "Inhaltes", eigentlich eine soziokulturelle Deutung, die nur für den deutschen Sprachraum gelten könnte. So heißt es für den oben genannten deutschen Spruch: "Sprichwort; gemeint ist hier Mund im Sinn von Mündel, Schutz."7

Wir kennen wirklich beim Wort Mund, geschrieben Munt, die Bedeutung Schutz, Fürsorge (auch heute ist in Zusammensetzungen und Ableitungen diese Bedeutung zu erkennen - Vormund, mündig); das Wort Mündel ist geblieben.

3 In: Karl Peltzer: Das treffende Zitat. Gedankengut aus drei Jahrtausenden. 4. erweiterte Ausgabe. Ott Verlag Thun und München 1957, S. 473.

4 Ebenda, S. 474.

5 Meyers Konversations-Lexikon. Fünfte Auflage. Leipzig und Wien. Bibliographisches Institut 1893. Zweiter Band, S. 187.

6 Deutsches Wörterbuch von Jacob und Wilhelm Grimm. München. DTV 1991. Band 12, S. 2586.

7 Elke Gerr: 4000 Sprichwörter und Zitate. München. Humboldt Taschenbuchverlag Jacobi KG 1989, S. 144 . 
$\mathrm{Da} B$ aber im lateinischen Spruch dieser Sinn enthalten ist, kann man bezweifeln. Vielleicht könnte man sagen, daß die Aussage alles einschließt, was den Geist und die Moral betrifft, und deswegen auch die oben angeführte Deutung der deutschen Variante möglich wäre.

Wolfgang Mieder führt in seinem Buch "Morgenstunde hat Gold im Munde" (es wird im ganzen Buch mit 150 Seiten nur dieser Spruch behandelt ${ }^{8}$ Richard Jente an, der behauptete, daß "keinerlei Beweise dafür vorlägen, daß das Sprichwort sich von einem etymologischen Schulmeisterwitz ableiten ließe."9

Mieder erwähnt weiter Jente, der doch glaubt, das deutsche Sprichwort sei aus dem lateinischen Sprichwort aurora musis amica abgeleitet. ${ }^{10}$ Natürlich gab es im Deutschen (so wie auch in vielen anderen Sprachen) Abweichungen, verschiedene eigenwillige Formulierungen, oft Parodien, die manchmal in den Bereich des Vulgären gerieten. Jente entdeckte auch einen frühen Beleg des lateinischen Sprichwortes aurora amica musis in Hermanni Germbergs Sprichwörtersammlung aus dem Jahre 1576. ${ }^{11}$

Den frühesten Beleg für das lateinische Sprichwort in der Form aurora musis amica fand jedoch Archer Taylor in einem Brief des Erasmus von Rotterdam aus dem Jahre 1497 an Christian Northaft in Lübeck, gedruckt zum ersten Mal in seinen Colloquies 1518.12

Das deutsche Sprichwort ist nach Friedrich Seiler ${ }^{13}$ zum ersten Mal in Michael Neanders Sprichwörtersammlung des Jahres 1585 zu finden. Auch Mieder stimmt Seiler zu und stellt fest, daß "sich nach etwa 100 Jahren die Version von 1585 (Die) Morgenstunde hat (das) Gold in Munde durchsetzen konnte. Dabei führt er wieder Jente an, der behauptet, daß das Wort "Mund(e)" um des Reimes willen mit Morgenstund $(e)$ in Verbindung gebracht wurde, auch wenn dies 'sinnwidrig' erscheinen muß ..."14

Man wollte das Syntagma Gold im Munde manchmal mit dem alten Brauch deuten, daß werte Geld- und Schmuckstücke bei einigen Völkern oft im Munde versteckt wurden.

Es handelt sich jedenfalls um ein Sprichwort, dessen Beliebtheit von Jahrhundert zu Jahrhundert wuchs. Es wurde auch sehr oft von den Schriftstellern verwendet. Im 19. Jahrhundert ist es besonders in vielerlei erzieherischen Texten zu finden.

8 Wolfgang Mieder: "Morgenstunde hat Gold im Munde". Studien und Belege zum populärsten deutschen Sprichwort. Wien. Verlag Edition Praesens 1997.

9 Richard Jente: Morgenstunde hat Gold im Munde. In: Publications of the Modern Language Associations, XXXXII (1927), S. $865-872$.

10 Siehe 8, S. 14.

11 Siehe 8, S. 14.

12 Archer Taylor, The Proverb; Cambridge/Mass. 1931, S. 48 - 49. Angeführt bei Mieder, S. 15.

13 Friedrich Seiler: Das deutsche Sprichwort. Straßburg 1918, S. 9 - 10.

14 Siehe 8, S. 14. 
Bei den Schriftstellen gab es bis in die allerjünste Zeit oft Umformulierungen, Andeutungen oder direkte Zitate in neuen Zusammenhängen, angepaßt dem literarischen Werk.

Auch die heutige Werbesprache macht von diesem Sprichwort immer wieder Gebrauch, oft mit Umdeutungen.

Der lateinische Spruch wird mit den Musen verbunden. Das waren bei den Griechen die Schutzgöttinnen des geistigen Lebens, besonders der Künste. Sie wurden als Schwestern im Gefolge des Apollon vorgestellt. Ursprünglich waren es drei, später neun, für die damals bekanntesten Künste: epische Dichtung (Kalliope); tragische Dichtung (Melpomene); komische Dichtung (Thalia); Lyrik (Euterpe); Chorlyrik und Tanz (Terpsichore); Liebesdichtung (Erato); Hymnendichtung (Polyhymnia); Geschichtsschreibung (Klio); Sternkunde (Urania).

Es handelte sich also beim Spruch vorwiegend um die geistige Arbeit. Die Eingebung, das geistige Schaffen war im Mittelmeerraum noch viel stärker an die Kühle der Morgenzeit gebunden. Aurora war ja auch die Göttin des Morgenrotes.

Aber es ist gewiß nicht nur die Kühle, die auf die Erfrischung des Geistes wirkt, sondern auch die Schönheit des Morgens, die dabei einen Einfluß ausübt.

Beides ist aber auch für eine physische Tätigkeit günstig, mag es sich um ein Wandern (damals ging man ja weite Strecken zu Fuß) oder um eine physische Arbeit im Gewerbe und auf dem Feld handeln.

Aus dem Mittelmeerraum stammt ja auch der Begriff Siesta (spanisch Mittagsruhe), da man in den heißen Mittagsstunden nicht arbeiten kann. Das Wort Siesta ist aus dem lateinischen (hora) sexta = "die sechste Stunde des Tages" abgeleitet, das heißt, nach dem Anbruch des Morgens. Dies zählte man nicht nach der heutigen Uhr.

Daß dieser Spruch für die menschliche Tätigkeit überhaupt gilt, obwohl er manchmal mehr mit der geistigen, manchmal mehr mit der physischen Arbeit verbunden wird, ist aus den soziokulturellen Anwendungen ersichtlich.

Am häufigsten kommt im heutigen Deutsch der Spruch Morgenstunde hat Gold im Munde vor.

Da haben wir einen Reim, und wenn man das Hilfsverb hat wegläßt, sogar ein Metrum: Morgenstunde - Gold im Munde.

Auf den ersten Blick scheint es, daß sich die deutsche Variante wirklich auch nur auf die geistige Tätigkeit bezieht.

In Wirklichkeit wird aber der Spruch sehr häufig im Volksmund verwendet, unter der Landbevölkerung, wo es viel physische Arbeit gibt.

Man will aber den Spruch immer wieder mit der geistigen Arbeit verbinden, sogar in Gesellschaftssystemen, wo die physische Arbeit hochgeschätzt wurde und wo die Arbeiterklasse die führende Rolle haben sollte. So hat man im Buch Deutsche 
Sprichwörter für Ausländer aus der ehemaligen DDR zum Spruch die folgende textuelle Erklärung (eine Veranschaulichung) geschrieben:

Am Morgen ist man am leistungsfähigsten.

Wie du das nur fertigbringst, Regina, jeden Morgen so zeitig aufzustehen und zu arbeiten! Ich bin immer froh, wenn die Vorlesungen nicht so zeitig beginnen und ich mich ausschlafen kann.

Morgens schaffe ich meine Arbeit am schnellsten. Probiere es doch auch einmal, und du wirst mir recht geben: Morgenstunde hat Gold im Munde. ${ }^{15}$

Durch den Kontrast schlechter - besser will man ein didaktisches Ziel erreichen. Es gibt also eine Textsorte mit Zielrichtung.

In der großen deutschen Zitatensammlung Das treffende Zitat ${ }^{16}$ findet man unter dem Stichwort Morgenstunde und bei der Übersetzung des lateinischen Sprichwortes mit Die Morgenstunde ist den Musen günstig noch zwei Hinweise auf Zitate unter den Stichwörtern Arbeit und beten.

Bei der Übersetzung des Originals von Hesiod wird das Wort Arbeit als Stichwort gebraucht. So ist es klar, daß die lateinische Version, die das Wort Arbeit nicht enthält, eine Anpassung der Aussage der römischen soziokulturellen Beziehung darstellt.

Wenn man in der direkten deutschen Übersetzung Hesiods nur die Schlüsselwörter in Betracht zieht, wird die Aussage noch klarer:

Das zentrale Wort ist gewiß auch bei Hesiod Morgenfrühe; die nächsten Schlüsselwörter wären Tagwerk, den Weg, die Arbeit. Es handelt sich doch um eine rege menschliche Tätigkeit im Leben, in dem Fall gebunden noch an eine gewisse Tageszeit, die wir auf unserer geographischen Breite als frisch kennen, meist verbunden auch mit der Ausgeschlafenheit.

$\mathrm{Da} ß$ aber mit all dem nur die physische Tätigkeit gemeint ist, kann man nicht behaupten. So sieht man auch aus den verschiedenen Übersetzungen verschiedene Aufnahmen, Auffassungen, auch Anpassungen des Sprichwortes an die jeweilige soziokulturelle Umgebung.

Die oben erwähnte deutsche Zitatensammlung hat beim Spruch Die Morgenstunde ist den Musen günstig noch einen Hinweis, wo auch eine Verbindung mit der Frömmigkeit ausgedrückt wird. So finden wir unter dem Stichwort beten das folgende Zitat: Ein guter Tag fängt an mit Gottes Preis; / es ist kein Geschäft so eilig als das Beten. - Schiller, Macbeth, II, 5 (Pförtner). ${ }^{17}$

$\mathrm{Ob}$ aus dem Macbeth oder bei Schiller wirklich zu entnehmen ist, daß es sich um eine Allusion an das Zitat aus Hesiod oder an den lateinischen Spruch oder an

15 Christa Frey, Annelies Herzog, Arthur Michel, Ruth Schütze: Deutsche Sprichwörter für Ausländer. Eine Auswahl mit Beispielen. VEB Verlag Enzyklopädie Leipzig 1970, S. 52.

16 Siehe 3, S. 474.

17 Siehe 3, S. 72. 
irgendeine Übersetzung handelt, kann man nicht beweisen. Jedenfalls kann man aber sagen, daß dieses Zitat je nach dem soziokulturellen Kontext verstanden, gedeutet und in der Übersetzung angepaßt wurde.

Die slowenische Variante ist sehr kurz, elliptisch. Sie lautet:

Rana ura - zlata ura.

("Frühe Stunde - goldene Stunde").

Wir haben da einen elliptischen Satz ohne Hilfsverb, außerdem besteht im Spruch ein ausgeprägtes Metrum, eine Gemination des Wortes $u r a$, was wie eine Epiphora wirkt und auch eine Betonung, eine Steigerung im Nennen der gewissen Zeit (Morgenzeit) ist. Es handelt sich weiters um das wiederholte Abstraktum ura mit zwei Attribuierungen - rana ura - zlata ura - was noch stärker wirkt. Die beiden Attribute könnten allein schon elliptisch alles sagen: rana (frühe) - zlata (goldene). Das gilt auch für das deutsche Kompositum Morgenstunde und für das Simplex Gold.

Der slowenische Spruch wird als Ellipse, wo das Hilfsverb fehlt, mit einem Gedankenstrich oder mit einem Beistrich geschrieben.

Interessant ist, daß im Buch Slovenski pravopis ("Slowenische Rechtschreibung") aus dem Jahr 1962 der Spruch unter dem Stichwort zlat ("golden") steht, im Buch Slovar slovenskega jezika ("Wörterbuch der slowenischen Sprache") aus dem Jahr 1994 aber unter dem Stichwort ran ("früh").

Logisch ist in solchen Büchern die alphabetische Reihenfolge der Stichwörter. Das sollte hier nach dem ersten Wort im Spruch wirklich ran(a) ("früh-e") sein. Warum man 1962 das Wort zlat(a) ("golden") als das zentrale Wort, nach dem der Spruch eingeordnet ist, empfunden hat, ist eine Frage. Die Rezeption, das Empfinden der gegliederten morphophonologischen und semantischen Struktur zeigt da wieder in einem gewissen Sinn den soziokulturellen Kontext, in dem Fall eines Autors oder einer Gruppe von Autoren.

Das viel ältere Wörterbuch Slovensko-nemški slovar ("Slowenisch-deutsches Wörterbuch") von Maks Pleteršnik aus dem Jahr 1895 (reproduzierter Nachdruck 1974) hat den Spruch auch unter dem Stichwort ran(a) ("früh") angeführt.

Der slowenische Spruch kann für jede Arbeit gültig sein. Weil aber das slowenische Volk jahrhundertelang überwiegend ein Bauernvolk war, wurde dieser Spruch am häufigsten unter der Bauernbevölkerung gebraucht, aber auch unter den Handwerkern und gleichfalls unter den slowenischen Städtern verschiedenen Berufes. Auch die Intellektuellen gebrauchen den Spruch bisweilen.

Warum das Bauernvolk diesen Spruch so liebte, obwohl er im Slowenischen nichts von der physischen Arbeit sagt, kann man hypothetisch auch so erklären:

Es gibt darin das Syntagma zlata ura ("goldene Stunde"). Das kann sich auf die Morgenröte (Aurora) und auf die aufsteigende Sonne beziehen. Es ist also auch etwas Ästhetisches, aber vor allem ist das die "goldene Zeit" für die Arbeit. Da darf man nichts versäumen, das ist den Menschen klar. Wir Slowenen haben auch den Spruch 
C̆as je zlato ("Zeit ist Gold") besonders in intellektuellen Kreisen zu einem Spruch Čas ni zlato, je več kot zlato ("Zeit ist kein Gold, sie ist mehr als Gold") erweitert.

Vom Beten ist in der slowenischen Variante Rana ura - zlata ura auf den ersten Blick keine Spur. Aber daß es ausgeschlossen ist, kann man nicht sagen. Fürs lange Beten gab es am Morgen auf dem Bauernhof keine Zeit. Man betete kurz beim Frühstück, später beim Morgenläuten, auch auf dem Feld. Am Abend, da betete man oft viel und lange.

Weil es sich schon bei Hesiod um eine didaktische Dichtung handelt und weil auch die lateinische Version des Spruches und die Versionen in vielen anderen Sprachen, darunter auch in der slowenischen, didaktische Züge der Aussage haben, ist es klar, daß der Spruch oft für erzieherische Ziele direkt verwendet wurde und wird. Oft wurde er aber in eine andere Dichtung entweder umgearbeitet oder klar alludierend aufgenommen. Ob dabei der Dichter den Spruch wirklich direkt im Sinne hatte oder nur in weiterer Erinnerung, oder ob er sogar nur eine allgemeine Aussage schrieb, die daran erinnert, weiß man oft nicht genau. Es gibt ja für das frühe Aufstehen und für die schon in der Frühe notwendige Arbeit auch andere Sprüche, vor allem aber praktische Hinweise und moralische Einstellungen, die anders oder ähnlich ausgedrückt werden können.

Der slowenische Bischof Anton Martin Slomšek (1800 - 1862), der 1859 die Diözese vom deutsch - österreichischen St. Adrä in Kärnten nach Maribor versetzte und der in dieser Stadt 1859 auch eine Theologische Hochschule gründete, war auch Dichter, Schriftsteller, Verfasser von Schulbüchern. Seine Gedichte sind hauptsächlich im Stil der Volkslieder und im Stil der didaktischen Dichtung geschrieben. Eins davon lautet verkürzt so:

Veselo jutro (1836)

Lepo jutro je svetlo sončece mene razsvetli; zarja jutranja mi veselje da $\checkmark$ šolo mi veli ... $\checkmark$ se na delo gre; mravlje, čbelice gredo živeč brat; ptičke mi pojo, rožice cveto, da grem $v$ šolo rad ... ${ }^{18}$

18 Aus: Knjiga o Slomšku. Zbornik ob stoletnici njegove smrti. Sestavila: Janez Poljanec in Franc Hrastelj. Celje. Mohorjeva družba 1962, S. 53. 
Fröhlicher Morgen (1836)

Schön der Morgen strahlt -

die helle Sonne wallt,

mich erhellt sie wohl;

's gibt das Morgenrot

mir Freude und Gebot,

daß ich zur Schule soll ...

Alles an die Arbeit geht;

Bienen und Ameisen steht

ihre Nahrung fern;

Vöglein singen mir,

Blümlein blühen mir,

zur Schule geh' ich gern ...

(Übersetzt von Mirko Križman)

Der Grundgedanke vom erwachenden Tag und vom Erwachen (im physiologischen und geistigen Sinn) des Menschen, der sich besinnt, daß er zur Arbeit (für die Kinder ist das die Schule) muß und die Pflichten am frischen, schönen Morgen mit allen himmlischen und atmosphärischen Erscheinungen auch mit Freude tut, ist aus dem Gedicht klar zu ersehen. Das Gedicht erinnert jedenfalls an den im Beitrag behandelten Spruch, es ist alles nur breiter, bildhafter, aber trotzdem auch knapp genug ausgedrückt. Eine solche Bildhaftigkeit kennt man auch in anderen Sprüchen und Volksliedern, obwohl die meisten Sprüche einen Gedanken, der auf vielen Erfahrungen beruht, prägnant, konzentriert ausdrücken. Aber wenn man vom Gehalt (Sujet) oder vom Thema eines Gedichtes spricht, kommt man auch zu einer knappen Äußerung darüber. In dieser Hinsicht ist das Gedicht doch dem behandelten Spruch ähnlich, mag es auch bildhafter und ausgearbeiteter sein.

Es gibt in der Literatur auch direkte Verwendungen des Sprichwortes:

Andreas Gryphius (1616 - 1664) hat in seinem Werk Horribilicribrifax im Kapitel Sempronius, wo er die lateinische und die deutsche Sprache kombiniert, den Spruch Aurora Musis amica verwendet.

Ferdinand Raimund (1790 - 1836) hat im Drama Der Verschwender (Philipp Reclam, 1967, S. 15.) die deutsche Form Morgenstund tragt Gold im Mund gebildet.

Bei Jeremias Gotthelf (1797 - 1854) findet man in der Erzählung Uli der Pächter die Form "... Golde, welches die Morgenstunde im Munde hat." (Sämtliche Werke in 24 Bänden. Erlenbach - Zürich. Eugen Rentsch, 1921, Bd. 11, S. 291 - 292.)

Karl Arenz (auch im 19. Jh.) schrieb ein zehnstrophiges Gedicht, wo die beiden letzten Zeilen jeder Strophe wie ein Refrain das Sprichwort "Die Morgenstund / Hat Gold im Mund" enthalten. ${ }^{19}$ 
Etliche Verwendungen, auch in verschiedenen abgeänderten Formen, kann man in der Literatur verschiedener Sprachen finden. Dasselbe gilt für besondere Intonationen, Anspielungen, Umdrehungen mit Parodie oder mit abgeänderten philosophischen und sozialen Pointen.

So finden wir bei Bertolt Brecht (Gesammelte Werke, hrsg. von Elisabeth Hauptmann, Frankfurt am Main: Suhrkamp, 1967, Bd. 8, S. 396) diese Form:

Ach, des Armen Morgenstund

Hat für den Reichen Gold im Mund ...20

Das ist eigentlich schon ein Übergang in eine gereimte Sentenz. Dasselbe gilt für die beiden folgenden Verse von Mascha Kaléko (1907 - 1975 - aus Das lyrische Stenogrammheft. Reinbek: Rowohlt, 1982, S. 43):

... Das mit der goldgeschmückten Morgenstunde

Hat sicher nur das Lesebuch erdacht ... ${ }^{21}$

$\mathrm{Da} ß$ man aus vielen Sprüchen Erweiterungen, Gegensprüche, Parodien gemacht hat, ist auch aus der Literatur- und Kulturgeschichte bekannt. Wir haben in diesem Beitrag schon die Erweiterung oder den Gegenspruch zum slowenischen Spruch Čas je zlato (Zeit ist Gold) erwähnt.

Es gehört zu den menschlichen Eigenschaften, daß sie die überkommenen Formulierungen lieben, daß ihre Gefühle mit großer Zähigkeit daran haften, oft wird aber "ein mißverständlich gewordener oder als widersinnig gewordener Spruch dadurch am Leben erhalten, daß die Phantasie ihn ergänzt oder an ihm weiterdichtet."22

Oft geht es aber um die Absicht, eine gewollte Burleske zu schaffen, namentlich wenn sie ihren Ursprung im Studentenwitz hat.

Die Scholaren haben z.B. den Spruch Morgenstund hat Gold im Mund als schulmeisterlich empfunden und haben dazu die Antithese gebildet: Faulheit stärkt die Glieder. $^{23}$

In Dr. Kokes Schlagworte des Humors. Ein Beitrag zur Kulturgeschichte unserer Zeit. Leipzig. Carl Reißner, 1991, S. 1 gibt es eine Umdrehung des Spruches: Morgenstunde ist aller Laster Anfang.

Bei Rudolf Rolfs finden wir in der Sammlung Inventur eines Hirns. Frankfurt am Main: Die Schmiere, 1970, S. 155 eine absichtlich triviale satirische Allusion in der Form Agitatorenstunde hat "Scheiße!" im Munde? Weiter wird durch die Abänderung des ursprünglichen Sprichwortes eine Allusion mit satirischer Zuspitzung bei Gerhard Uhlenbeck erreicht. In seinen Medizinischen Aphorismen (Heidelberg: Jungjohann, 1982, S. 69) gibt es diese Form: Private Zahnsprechstund' hat Gold im Mund. ${ }^{24}$

21 Ebenda, S. 112.

22 Werner Krauss: Die Welt im spanischen Sprichwort. Verlag Philipp Reclam jun. Leipzig 1975. Einleitung, S. 24.

23 Ebenda, S. 25. 


\section{Breitere Aussagen und Knappheit als Variationen und $\ddot{U} b$ ersetzungsprobleme}

Besonders die Übersetzungen von Textsorten, die auf mehreren Konnotationen beruhen, sind eine "Auseinandersetzung zwischen dem Menschen und dem (literarischen) Gegenstand; hier fängt schon die Verwandlung an, die der Text erfährt, nicht erst bei der sprachlichen Erfahrung." 25

Ein schönes Beispiel, wie die Sentenz, die in der heutigen knappen Fassung Irren ist menschlich (Errare humanum est) bekannt ist, in der Zeit und bei verschiedenen Völkern sowie bei verschiedenen Autoren variiert, werden wir hier ebenfalls kurz zeigen.

Der Gedanke ist griechischen Ursprungs, man kann ihn aus Theognis, Elegeia 327 f. ableiten: "Denn Verfehlungen folgen den Menschen, den sterblichen, auf dem Fuße". In Sophokles' Antigone gibt es den folgenden Satz: "Denn den Menschen, ihnen allen, ist gemeinsam, daß sie sich verfehlen".

Bei Menander, Phanion, Fragment 432 Körte heißt es: "Als ein Mensch, der ich bin, habe ich mich verfehlt - kein Grund, sich darüber zu verwundern."

Mit der griechischen Komödie kommt der Gedanke nach Rom. Bei Terenz, Brüder 579, heißt es: "Censen hominem me esse? Erravi." (Glaubst du, daß ich ein Mensch bin? Ich habe mich geirrt).

Plinius der Jüngere hat in seinen Briefen 9, 12 die folgende Äußerung: "Non omnes homines aliquo errore ducuntur?" (Lassen sich nicht alle Menschen von irgendeinem Irrtum leiten? $)^{26}$

Ähnliche Beispiele kann man noch finden.

Der Gedanke lebte im Mittelalter weiter. So gibt es ihn z.B. bei Hieronymus, Briefe $57,12:$ :... quia et errasse humanum est et confiteri errorem prudentis," ... (... weil sowohl geirrt zu haben menschlich ist als auch den Irrtum einzugestehen klug ...")

Augustin sagt in seinen Predigten 164, 10, 14 (in Migne, Patrologia Latina, Band 38, Spalte 901 f.) folgendermaßen: "Humanum fuit errare, diabolicum est per animositatem in errare manere." (Menschlich war es zu irren, teuflisch ist es, leidenschaftlich im Irrtum zu verharren. $)^{27}$

In den heutigen Wörterbüchern für die breiteren Kreise der Studierenden finden wir meist die knappe Form der Übersetzung Irren ist menschlich. In einsprachigen

24 Die letzten Beispiele sind ebenso im Werk von Wolfgang Mieder "Morgenstunde hat Gold im Munde" angeführt. Siehe 8, S. 123 ff.

25 Fritz Güttinger: Zielsprache. Theorie und Technik des Übersetzens. Zürich. Manesse Verlag. Conzett - Huber 1963, S. 42.

26 Alle Zitate und Übersetzungen aus: Veni, vidi, vici. Geflügelte Worte aus dem Griechischen und Lateinischen. Ausgewählt und erläutert von Klaus Bartels. München. Deutscher Taschenbuch Verlag 1992, S. 72 f. 
Wörterbüchern ist die muttersprachliche Form an erster Stelle. Oft ist die lateinische Form überhaupt nicht angegeben, wie z.B. im Deutschen Wörterbuch von Gerhard Wahrig 1986, im Wörterbuch der deutschen Gegenwartsprache 1984, im Deutschen Universalwörterbuch (Duden) 1989. Das Bedeutungswörterbuch von der Serie Der Große Duden hat die Sentenz nicht, auch keinen Vers. Wohl gibt aber das Stilwörterbuch (1963) einige Nuancierungen und Erweiterungen an: Irret euch nicht, Gott läßt sich nicht spotten. (bibl.) Irren ist menschlich. Mancher hat, aus Furcht zu irren, sich verirrt (Lessing). Sobald man spricht, beginnt man schon zu irren. (Goethe). Es irrt der Mensch, solang er strebt. (Goethe).

Es ist interessant, das eine sehr alte Ausgabe des Stilwörterbuches vom Großen Duden (aus dem Jahr 1937) nicht nur alle Zitate aus dem modernen Duden -Stilwörterbuch enthält (auch das Zitat aus der Bibel), sondern noch ein zusätzliches, das man auch als Allusion auf die Moral der (damaligen) Zeit verstehen könnte: Durch Heftigkeit ersetzt der Irrende, was ihm an Wahrheit und an Kräften fehlt. (Goethe). Es ist aber eigentlich nur ein Zitat mit einem kritischen Gedanken für alle Zeiten.

Einige von den Zitaten sind nur im breiteren Zusammenhang mit der knappen Form, die wir besprechen, zu verstehen. Aber sie zeugen doch davon, wie das Irren den menschlichen Geist aller Kulturen beschäftigte, auch davon, wie die übernommenen Erfahrungen und Äußerungen modifiziert, erweitert, in der Form gekürzt oder zusätzlich, manchmal aufs Neue bearbeitet wurden. Auch wählte man manchmal ein Zitat und übersetzte es nicht wörtlich, sondern schon mit einer Neubildung, entweder einer übernommenen oder zusätzlichen Form. Je nach dem Wissen des Autors, je nach seiner Bestrebung, nach seinem stilistischen Gefühl oder nach dem Bedarf des Buches.

So findem wir im Buch Slovar slovenskega knjižnega jezika (Wörterbuch der slowenischen Standardsprache) aus dem Jahr 1994 nach der Bemerkung ekspr. (ekspresivno - "expressiv") nur die Prägung Človeška glava se lahko moti ("der menschliche Kopf kann sich irren"). Dagegen ist im Buch Slovar tujk ("Fremdwörterbuch") von France Verbinc (1958) das Original errare humanum est mit der Übersetzung motiti se je človeško (Irren ist menschlich), aber mit einer zusätzlichen Erklärung vsakdo se lahko zmoti ("Jeder kann sich irren") und mit der Angabe des Autors dieser letzten Aussage (Seneka).

Silva Trdina hat in Ihrem umfangreichen Buch Besedna umetnost (Wortkunst) aus dem Jahr 1958 eine Reihe von Zitaten gesammelt, die nach ihrer Meinung wichtig sind und die jeder gebildete Mensch kennen muß. Da hat sie auch die Sentenz Errare humanum est und die "Übersetzung" Človek se moti, dokler živi. (Der Mensch irrt, so lange er lebt.) Das ist aber keine wörtliche Übersetzung, die wir kennen, sondern schon der modifizierte Vers aus Goethes Faust (Prolog im Himmel: ... Es irrt der Mensch, solang er strebt.)

Auch keine genaue Angabe des Verses von Goethe hat Silva Trdina gewählt. Es war wahrscheinlich der Klang und der Wert der klassischen Dichtung (die Autorin war klassisch erzogen und sehr gebildet) maßgebend. Ein starkes Gefühl, ein Gedächtnis und auch eine ästhetische Bestrebung waren dabei beteiligt. 
Es könnten noch einige weitere Beispiele der Übernahme, der soziokulturellen Anpassung, der absichtlichen Modifizierung und Erweiterung von sog. Kurzformen der Textsorten gezeigt werden. Natürlich gibt es auch unabhängige Ähnlichkeiten. In diesem Beitrag mußte der Autor eine Auswahl treffen.

$$
S c h l u \beta
$$

Es handelt sich nicht immer nur um die Auffassung in einem einzelnen Kopf, sondern in einer soziokulturellen Gemeinschaft, auch im weitesten Sinne, bezogen auf eine historische Epoche. So können z.B. Sprüche, die von einem Individuum übersetzt wurden, Fuß fassen oder nicht. Wir kennen bei verschiedenen Nationen oder sozialen Gruppen Sprüche, die man in anderen Ländern nicht kennt. Dagegen sind viele allen oder den meisten Völkern bekannt. Daß es aber oft zu Nuancen oder Abänderungen kam, ist eine natürliche Erscheinung. Parodien vieler Art gab es immer.

Nicht alle Gründe, die auf den menschlichen Geist wirken, kann man feststellen, einige aber doch. Vergleiche mit den Sprach- und Literaturkenntnissen eines Verfassers oder Übersetzers, das Einleben und Kennen seiner mentalen Eigenschaften, der historisch bedingten materiellen und geistigen sowie der damit verbundenen kulturellen Besonderheiten, kann man bis zu einem bestimmten Grad ergründen. Ebenso welche Ähnlichkeiten und Unterschiede auftreten, zum Teil auch warum. All das läßt doch Forschungen zu, die sprachliche und etliche breiter zusammenhängende Feststellungen ermöglichen. Für die interkulturellen Sichten bei den kurzen Textsorten ist das wichtig. Besonders die ideologischen Grundlagen und Bestrebungen, auch nur Teile davon, eingebaut in Kontexte verschiedener Art, muß man berücksichtigen.

Hierher gehören auch die didaktischen Bestrebungen.

Verschiedene Kulturen sind nicht nur die verschiedenen Völker, die verschiedenen Religionen und die verschiedenen Literaturen, sondern auch das materielle und geistige Leben der Individuen sowie der politischen, kulturellen und wissenschaftlichen Gruppen. Man kann immer von den einzelnen soziokulturellen Umgebungen in Zeit und Raum sprechen.

Ein Individuum ist nie isoliert, es ist immer Teil eines sozialen und ethnischen, sprachlichen und kulturellen Gebildes, das jeden Menschen prägt und ihm auch Möglichkeiten gibt. Gleichzeitig reagiert aber auch das Individuum aus sich mit seinen Fähigkeiten und Bestrebungen. So wirkt es auf die Umgebung. Da zeigt sich die Beziehung zur Überlieferung. Die Einflüsse sind immer vielseitig.

Übersetzungen erweitern die Einsicht in die Überlieferung, jener der Muttersprache und jener der Fremdsprachen. Dabei geht es nicht nur um die Konfrontation der Sprachen, sondern auch der soziokulturellen Umgebungen und des schöpferischen Vermögens. Affinitäten, Ablehnungen oder Anpassungen verschiedener Art sind oft einfachere, oft aber komplexere Erscheinungen. 


\section{Povzetek \\ PREGOVORI, SENTENCE, CITATI KOT KRATKE OBLIKE ALI "PREPROSTE OBLIKE" BESEDILNIH VRST Z MEDKULTURNEGA VIDIKA}

Zlasti za pregovore in reke (le-ti izražajo delno veljavne življenjske resnice) lahko ugotovimo nekatere pojave, ki sodijo na področje jezikoslovja, in nekaj detajlov stilistike, tudi s stališča estetike.

- Za razumevanje, tudi za konotacijo, je treba poznati semantiko in logično slovnico celokupnega jezika, $\mathbf{v}$ katerem se te kratke oblike besedilnih vrst pojavljajo; če je potrebno, se je treba ukvarjati tudi z variantami (diasistemi) kakega jezika. Logična slovnica se ne ponuši, ampak izraža nove ali dodatne odnose. Tako se npr. pragmatična prislovna določila časa in kraja prenesejo s pragmatičnega področja na splošno veljavnost.

- Če primerjamo pregovore in reke medkulturno, ni dovolj, da poznamo le slovnico jezikov, iz katerih so vzeti, potrebni so odtenki v semantiki glede duha konteksta. Le-ta ni samo jezikoven, ampak tudi individualen in socialnokulturen, izžareva duha individua, okolja in epohe.

- Treba je upoštevati, da so mnogi slovniški in semantični pojavi $v$ takih kratkih besedilnih vrstah povezani še z glasoslovnimi pojavi, saj gre pogostokrat za ustno izročilo in ustno komuniciranje.

- Pri prevajanju je treba upoštevati jezikoslovne in retorične prvine, paziti moramo, da dobimo skupaj s temi prvinami tudi ustrezno intelektualno ter estetsko vrednost.

Prispevek obravnava pregovor rana ura - zlata ura (aurora musis amica) in omenja izvor misli pri Hesiodu, latinsko tvorbo ter prve vire $\mathrm{v}$ nemščini (16. stoletje). Nato prikazuje prispevek modifikacije pregovora vse do današnjih dni. Uporabljali so jih različni avtorji, bodisi za slovarsko gradivo, za zbirke pregovorov in rekov ali pa kot citate v literarnih delih. Zlasti v 19. stoletju so po uvedbi splošne šolske obveznosti omenjeni pregovor zelo pogostokrat uporabljali za vzgojne namene. Tudi v književnih delih, ki so vsebovala bolj ali manj vidne vzgojne tendence, najdemo izvirne citate ali razpoznavne predelave tega pregovora. Tudi A. M. Slomšek je jasno vkomponiral misel iz tega pregovora v pesem Veselo jutro.

Izjemno popularnost je ohranil pregovor tudi v nemški književnosti 19. in 20. stoletja. Poznamo direktne navedbe, različne aluzije in mnoga parodiranja. Vse omenjeno s pridom uporablja tudi najsodobnejši jezik reklam, zlasti pa jezik moderne satire na različna početja gospodarstvenikov, politikov in ljudi raznovrstnih poklicev ter značajev.

Veliko parodij so ustvarili že sholarji, v novejšem času komediografi in satiriki, tudi različni družbeni kritiki $v$ literarni obliki (Hermann Kant, Mascha Kaléko, B. Brecht, K. Zuckmayer, Ulrich Erckenbrecht in drugi).

$V$ prispevku je nadalje obdelan izrek errare humanum est (motiti se je človeško). Pisno in ustno je uporabljen izredno pogosto. Vendar pa $v$ taki kratki obliki ne izvira iz antike. Misel pa seveda najdemo živahno niansirano že pri grških in rimskih avtorjih in se nadaljuje $\mathrm{z}$ različnimi modifikacijami skozi srednji in novi vek.

Najbolj znan je Goethejev verz iz Fausta (Prolog v nebesih) Es irrt der Mensch, solang er strebt (dobeseden prevod ali eden izmed možnih prevodov bi bil: človek se moti, dokler stremi).

Avtor prispevka je izbral več zbirk citatov, tudi več splošnih slovarjev, slovarjev tujk, enciklopedij itd., ki navajajo sentenco. Le-ta je prešla v različnih oblikah v reke in pregovore ter pesniške podobe. Pri tem je včasih na prvem mestu latinska oblika, včasih domača. Prevodi pa niso enotni. Opazimo, da gre kdaj za dobeseden prevod, kdaj za modifikacije glede na znanje in okus avtorja (avtorjev). Nameni so modrovanja, pridigarstvo, psihologiziranja. Nemalokrat pozvanja v prevodu Goethejev verz; s tem je hotel kakšen avtor pokazati izobrazbo ali pesniško žilico.

Različne kulture niso samo različni narodi, različne religije, različna literarna dela, ampak tudi materialna in duhovna stremljenja posameznikov in kulturnih ter znanstvenih skupin pa njih znanja in stremljenja. Seveda lahko govorimo o posameznih sociokulturnih okoljih v času in prostoru. Posameznik ni nikoli izoliran, ampak je vedno del socialne in etnične, jezikovne in kulturne tvorbe, ki mu daje pečat in možnosti. Hkrati se pa tudi posameznik odziva s svojimi individualnimi zmožnostmi in hotenji na okolje. Sem sodi tudi odnos do izročila. Vplivi so seveda mnogostranski. Prevodi niso samo konfrontacija jezikov, ampak tudi sociokulturnih okolij in ustvarjalnih sposobnosti. Afinitete, odklonitve ali prilagoditve so včasih preprostejši, včasih pa bolj kompleksni pojavi. 